\title{
Les phrases nominales événementielles exprimant une valeur affective
}

\author{
Makoto Kaneko \\ Université d'Okayama (Japon) \\ kaneko06@cc.okayama-u.ac.jp
}

\section{Introduction}

Cette étude ${ }^{1}$ a pour but de rendre compte de la façon dont les phrases nominales comme (1a,b) expriment une « valeur fortement affective de la phrase (étonnement, regret, vive opposition, etc) $»^{2}$ (Le Bidois \& Le Bidois, 1971 : 379), ou « un désaccord ou contraste avec une situation ou un fait présent » (Sandfeld, 1965 : 156). (1a) exprime ainsi, selon le contexte, l'étonnement, le regret ou le désaccord. (1b) illustre le cas de vive opposition ou de contraste avec le discours précédent, analogue au contraste exprimé par le conjonctif concessif quoique en (2) :

(1)a. Ah! Mon dieu ! dit-il, Monsieur Michel qui est mort! (Sandfeld, $1965: 155$ )

b. Nous sommes à la gare, déjà ! Moi qui avais encore tant de choses à vous conter. (idem.156)

(2) Quoiqu'il pleuve, Jean part.

L'argumentation s'appuie sur une comparaison avec la particule de focus nante du japonais, comme en $(3 \mathrm{a}, \mathrm{b})$. La particule nante est attachée à une phrase entière en (3a) et à un NP en $(3 \mathrm{~b})^{3}$ :

(3)a. Paul ga nakunat-ta nante !

Paul Nom mourir-Pst NANTE

'Paul est mort, ce n'est pas possible / je ne peux pas le croire !'

b. Paul wa nihongo nante eran- da.

Paul Top japonais NANTE choisir-Pst

'Paul a choisi (ex. comme langue étrangère à apprendre à l'école) une langue comme le japonais (et non pas l'espagnol ou l'allemand) !'

La particule nante exprime une réaction affective du locuteur. En empruntant la caractérisation de Numata $(2000)^{4}$, nante en (3b) suggère que le japonais ne fait, selon le locuteur, pas partie des langues que Paul devrait naturellement choisir d'étudier à l'école. De même, la même particule en (3a) suggère que la mort de Paul ne fait pas partie des événements qui devraient se produire selon les prévisions du locuteur, ou selon le déroulement naturel des choses.

Il est à noter que la particule nante est composée étymologiquement du mot WH nani ('quoi') et du complémenteur spécialisé pour la ciation te. Yamanaka (2007) observe que, malgré la grammaticalisation entraînant la contraction phonologique et l'intégration morphologique de ces deux composantes, la sémantique de citation y subsiste : cet auteur montre en effet que la particule nante sert dans la plupart des cas à reprendre l'information qui vient d'être transmise par les autres.

Je caractériserai la sémantique de nante en (3a,b) en la comparant avec les questions de reprise exprimant l'étonnement en $(4 \mathrm{a}, \mathrm{b})$, qui impliquent le mot WH nani et le complémenteur de citation te. Je gloserai celui-ci par 'vous dites' pour mettre en relief la sémantique de ciation :

(4)a.

$$
\begin{aligned}
& \text { A : Paul ga nakunat-ta. - B : nan- dat- te ? } \\
& \quad \text { Paul Nom mourir-Pst -- } \text { quoi-Cop }{ }^{5} \text {-vous dites? }
\end{aligned}
$$


'A : Paul est mort. - B : Que dites-vous ?'

b. A : Paul wa nihongo o eran-da. - B : Paul ga nani o eran- da(-dat)- te. Paul Top japonais Acc choisir-Pst Paul Nom quoi Acc choisir-Pst-Cop-vous dites

'A : Paul a choisi le japonais. - B : Vous dites que Paul a choisi quoi ?'

Je rapprocherai par ailleurs les phrases nominales de type (1a) des questions de reprise exprimant l'étonnement comme en $(5 \mathrm{a}, \mathrm{b})$ :

(5)a. A : Paul est mort. - B : Qui est mort ?

b. A : Paul est mort. - B : Quoi ?

Il est aussi à noter que nante sert d'adverbe exclamatif, comme en (6a), et que l'exclamation peut être exprimée en français par les phrases nominales, comme en $(6 b)^{6}$ :

(6)a. nante kireena hana!

NANTE joli fleur

'Quelle jolie fleur!'

b. Les beaux biceps que tu as !

Dans ce qui suit, j'avancerai trois hypothèses principales : (i) les phrases nominales comme (1a,b) ne sont, de même que la phrase nominale en $(6 \mathrm{~b})$, pas des phrases elliptiques dont le thème ou le rhème sont contextuellement récupérés, mais des phrases indépendantes dont l'ensemble est focalisé ; (ii) en vertu de leur structure de focus, elles donnent lieu à la proposition focalisée et à l'ensemble des propositions contrastées qui correspondent respectivement à la proposition citée et aux questions de reprises en $(5 \mathrm{a}, \mathrm{b})$; (iii) la valeur affective des phrases nominales est une implicature conventionnelle dérivée d'une condition imposée sur l'emploi approprié des questions de reprise, selon laquelle la proposition citée n'est pas vraie dans le savoir ou la croyance du locuteur.

La discussion sera suivie dans l'ordre suivant : je passerai d'abord en revue les trois principaux travaux existants sur les phrases nominales, pour choisir comme point de départ celui de Portner \& Zanuttini (2005) $(\S 2)$; je préciserai ensuite la sémantique de la particule de focus nante en la comparant avec les questions de reprise $(\S 3)$; j'avancerai enfin, en appliquant lea résultata de la section 3 aux phrases nominales de type $(1 \mathrm{a}, \mathrm{b})$, des hypothèses sur leur syntaxe et leur sémantique, et présenterai certains arguments qui les appuient. L'analyse proposée permettra par ailleurs de saisir un point commun entre la phrase nominale exprimant un contraste comme (1b) et le conjonctif concessif quoique incluant l'opérateur WH quoi comme en (2) (§ 4). La section 5 récapiturera les résultats de cette étude.

\section{Travaux antérieurs sur les phrases nominales}

\subsection{Omission du Thème spatio-temporel}

Les phrases nominales du français comme (1a,b) sont analysées par Sasse (1987) et Furukawa (1996), entre autres, comme «serv[ant] à présenter un événement comme un bloc 'non structuré' 》 (Furukawa, 1996 : 62), c'est-à-dire un jugement thétique dépourvu de dissociation Thème-Rhème. J'appellerai ces phrases nominales phrases nominales événementielles en les distinguant des phrases nominales exprimant le haut degré comme (6b) que j'appellerai phrases nominales exclamatives. Parmi les travaux antérieurs traitant des phrases nominales événementielles, Lefeuvre (1999 : 285-288) soutient que ces phrases averbales « répondent à la question Qu'est-ce qu'il y a ? Qu'est-ce qui se passe ? Qu'est-ce qui arrive ? " et impliquent, malgré l'apparence, une mise en relation par la modalité entre le thème et le rhème : (7a) correspond au rhème dont le thème implicite, de nature spatio-temporelle, est récupéré par la situation d'énonciation, comme le schématise $(7 b)$ : 
(7)a. Le lavabo qui déborde! (Lefeuvre, $1999: 287$ )

b. [thème (ici et maintenant)] [rhème le lavabo qui déborde]

c. C'est le lavabo qui déborde !

(8) Que se passe-t-il ? - Un couple qui vient de sortir du magasin... (Simenon, G. Le chien jaune)

Cette analyse se réduit à supposer le prédicat principal implicite c'est en (7c), dont le sujet pronominal ce reprend le thème spatio-temporel. Erteschik-Shir (1997) et Jäger (2001) soutiennent également que les phrases thétiques disposent d'un argument implicite spatio-temporel ${ }^{7}$. Il y a certes des cas où le prédicat pricipal c'est ellipsé est naturellement récupéré, comme en (8). Ces cas ne manifestent toutefois pas de valeur affective. L'analyse du thème elliptique ne rend donc pas compte de celle-ci.

\subsection{Omission du rhème exprimant une valeur affective}

D'autre part, en discutant les phrases nominales exclamatives illustrées par (6b) et (9a), Michaelis \& Lambrecht $(1996: 244)$ avancent l'idée que ce sont des «phrases elliptiques correspondant au thème dont le rhème implicite exprimant un jugement affectif est pragmatiquement inféré ${ }^{8}:$ (9a) est donc paraphrasé par (9b) et schématisé par $(9 c)$ :

(9)a. The amount I spent ! (Michaelis \& Lambrecht, $1996: 244$ )

b. It's amazing the amount I spent! (ibid.)

[extraposition nominale]

c. [thème the amount I spent] [rhème (is surprising)]

Cette analyse rend directement compte de la valeur affective, mais nécessisterait différents types de prédicats affectifs pour exprimer diverses valeurs affectives comme, " étonnement, regret, vive opposition, etc. » (Le Bidois \& Le Bidois, 1971: 379). De plus, il semble difficile de trouver un prédicat susceptible d'exprimer un contraste avec le discours précédent comme en (1b).

\subsection{Aucune omission : analyse comme une phrase indépendante}

Pour les phrase nominales exclamatives comme en (10a), Portner \& Zanuttini (2005) soutiennent i) que tout en étant construites du NP et de la relative, elles ne sont pas elliptiques mais indépendantes, et que ii) leur sémantique est équivalente à celle des phrases exclamatives incluant un mot $\mathrm{WH}$, quels en (10b) ou que en $(10 \mathrm{c})$ :

(10)a. Les beaux biceps que tu as !

b. Quels beaux biceps tu as !

c. Qu'est-ce que tu as de beaux biceps !

En faveur de l'hypothèse de la nature phrastique, ces auteurs invoquent le fait que la reprise pronominale ne montre pas les accords de genre et de nombre avec le NP tête, comme en (11a) et (11b):

(11)a. *Ils sont fous les beaux biceps que tu as !

b. C'est fou les beaux biceps que tu as !

Pour confirmer la nature exclamative, Portner \& Zanuttini (2005) observent que cette construction manifeste la factivité couramment observée pour les phrases exclamatives. Ainsi, on ne peut pas l'enchâsser sous une principale qui en modifierait la valeur de vérité, comme en (12b) :

(12)a. Je sais \{quels beaux biceps tu as / les beaux biceps que tu as\}.

b. *Je ne sais pas \{quels beaux biceps tu as / les beaux biceps tu as\}. 
Du côté sémantique, Zanuttini \& Portner (2003) soutiennent que l'exclamative WH comme (10b,c) dénote, exactement comme une question $\mathrm{WH}$, un ensemble de propositions. Ces auteurs définissent en outre sa sémantique non seulement par la factivité, mais aussi par la condition d'élargissement ('widening') : en énonçant une exclamation, le locuteur cherche, dans le domaine initial (D1) consistant en membres contextuellement pertinents, le membre effectivement observé dans la situation actuelle. Cette opération de parcours est effectuée par le liage de la variable de la part de l'opérateur WH. Mais le membre observé dans le monde actuel n'est pas trouvé dans le domaine initial, qui doit donc être élargi en un nouveau domaine (D2) ${ }^{9}$. Si l'on représente, par x et $\mathrm{y}$, la variable de degré de la beauté en D1 et celle dans la partie complémentaire de D1 en D2, la sémantique de (10b,c) peut être formalisée par (13a,b). En mots, (13b) indique que le degré y est supérieur à tous les degrés x en D1 :

(13) sémantique de l'exclamation

a. $\quad \mathrm{w} 0 \in \mathrm{p}=\lambda \mathrm{w}(\exists \mathrm{y}($ tu as de beaux biceps de degré $y$ en $\mathrm{w}))$

[factivité]

b. $\forall \mathrm{x} \forall \mathrm{y}[\mathrm{x} \in \mathrm{D} 1 \wedge \mathrm{y} \in(\mathrm{D} 2-\mathrm{D} 1) \rightarrow \mathrm{x}<\mathrm{y}]\} \quad\left[\mathrm{D} 1\right.$ est le domaine intial et D2 est le domaine élargi ${ }^{10}$

[condition d'élargissement]

Du point de vue syntaxique, dans l'exclamative WH, l'opérateur qui effectue le parcours de variable est fourni par un mot WH explicite comme quels ou que en (10b,c). Selon Portner \& Zanuttini (2005), dans les phrases nominales exclamatives comme en (10a), c'est la syntaxe de relative qui met en oeuvre l'opérateur WH implicite au moyen du mouvement WH, comme en (14) :

$$
\text { syntaxe des phrases nominales exclamatives }
$$$$
\text { [DP les [CP beaux biceps-Wh Op } \left.\left.\text { k }_{\mathrm{C}} \text { que }\left[\text { tu as } \mathrm{t}_{\mathrm{k}}\right]\right]\right]^{11}
$$

Cette analyse permet de rendre compte du sens exclamatif des phrases nominales sans supposer aucune omission. Mais il faudrait préciser comment la factivité et la dénotation de l'ensemble de propositions arrivent à transmettre la condition d'élargissement en (13b). En outre, la notion du domaine initial ne permet pas à elle seule de saisir la diversité des valeurs affectives (ex. étonnement, regret, vive opposition, etc. ) exprimées par les phrases nominales événementielles.

\section{Particule de focus nante}

En visant à compléter l'analyse des phrases exclamatives par Zanuttini \& Portner (2003), cette section comparera la sémantique de la particule nante avec celle des questions de reprise. Je commencerai par présenter l'analyse des questions de reprise par Ginzburg \& Sag (2000) (§ 3.1). Je réinterpréterai ensuite la sémantique de cette construction en terms du focus ( $\$ 3.2$ ). Puis, j'envisagerai un point commun et une différence entre les questions de reprise et la particule nante (§ 3.3). La diversité des valeurs affectives sera saisies en me référant aux travaux antérieurs sur la modalité ( $\$ 3.4)$. Je proposerai enfin d'analyser les valeurs affectives de nante comme des implicatures conventionnelles ( $\$ 3.5$ ), avant d'appliquer les résultats de la section 3 à l'analyse des phrases nominales événementielles du français dans la section 4.

\subsection{Questions de reprise}

Ginzburg \& Sag (2000) examinent les questions de reprises comme en (15a) dans l'optique dialogique. Selon ces auteurs, la question de reprise en (15aB) est paraphrasée par (15bB), qui inclut l'expression de citation « vous venez de dire », et montre que le locuteur a du mal à ancrer dans le fonds commun ('common ground') l'information qui vient d'être transmise :

(15)a. A : J'aime Vyachoslav Voinovich. - B : Tu aimes QUI ? (Ginzburg \& Sag, 2000 : 258)

b. B : Qui est-ce que vous venez de dire que vous aimez ? (idem.259) 
Ces auteurs distinguent de plus trois cas de figure : (i) question écho, où le locuteur $\mathrm{B}$ n'a pas bien entendu une partie de l'énoncé de l'interlocuteur $\mathrm{A}$, par exemple, le nom propre difficile à retenir Vyachoslav Voinovich en (15a); (ii) question sur le référent, où le locuteur n'arrive pas à identifier le référent en cause. Dans ce cas, l'interlocuteur A continuera par exemple en répondant « Le Russe que vous avez vu à la soirée. »; (iii) expression d'étonnement ('expression of astonishment') selon laquelle " le locuteur a été étonné de ce que l'allocutaire venait de lui dire. Il lui a donc demandé à qui il avait dit qu'il aimait $»^{12}$ (Ginzburg \& Sag, $2000: 260$ )

Ces trois cas sont paraphrasés par $(16 a, b, c)$. Ces paraphrases montrent que dans la situation où une proposition vient d'être affirmée, la mise en question de la même proposition n'est discursivement appropriée que si cette question présuppose une certaine négation :

(16)a. Je n'ai pas entendu le nom que vous venez dire. Pouvez-vous le répéter? [question écho]

b. Je ne peux pas idenfier la personne dont vous venez de parler, c'est-à-dire, Vyachoslav Voinovich. Pouvez-vous me donner une description de cette personne? [question sur le référent]

c. Je ne peux pas croire ce que vous venez de dire, c'est-à-dire, que c'est Vyachoslav Voinovich que vous aimez. Pouvez-vous le confirmer?

[expression d'étonnement]

Ginzburg (à paraître) distingue par ailleurs l'état informationnel de chacun des participants au dialogue en deux parties, à savoir la partie publique et la partie privée, comme le schématise $(17)^{13}$ :

(17) [État Informationnel [Public (....fonds partagé.....)] [Privé (... fonds en arrière-plan)]]

(adapté de Marandin, $2005: 51-53$ )

Corrélativement, cet auteur distingue deux types de fonds : fonds partagé en commun entre les interlocuteurs qui se trouvent dans la partie publique ; fonds en arrière-plan dans la partie privée, qui correspond à « la description du but du locuteur et la description du savoir [ou de la croyance] qu'il détient indépendamment en propre à chacun des tours » (Marandin, $2005: 53$ ).

Cette distinction implique que la question de reprise exprimant l'étonnement en (15aB) (i) exige la condition d'appropriation selon laquelle la proposition citée « A aime Vyachoslav Voinovich » n'est pas vraie dans le fonds privé en arrière-plan du locuteur B et (ii) sert à demander à l'interlocuteur $\mathrm{A}$ de confirmer que la même proposition devrait être traitée malgré tout comme vraie dans le fonds partagé, comme le représentent $(18 \mathrm{a}, \mathrm{b})$ :

(18)a. $\quad[[$ A aime Vyachoslav Voinovich $]]=0$ dans le fonds privé de $\mathrm{B}$

[condition d'appropriation]

b. $\quad[[$ A aime Vyachoslav Voinovich $]]=1$ ou 0 dans le fonds partagé

[demande de confirmation]

\subsection{Questions de reprise et focus}

Les questions de reprise du japonais en (19a,b) peuvent également exprimer l'étonnement :

(19)a. A : Paul ga nakunat-ta. - B : nan-dat-te ? (=(4a))

Paul Nom mourir-Pst -- quoi-Cop-vous dites?

'A : Paul est mort. - B : Que dites-vous?'

b. A : Paul wa nihongo o eran- da. - B : Paul ga nani o eran- da(-dat)-te. $(=(4 b))$

Paul Top japonais Acc choisir-Pst Paul Nom quoi Acc choisir-Pst-Cop-vous dites

'A : Paul a choisi le japonais. - B : Vous dites que Paul a choisi quoi ?'

L'étonnment est aussi exprimé par les questions de reprise 'littérales', pour parler comme Ginzburg \& Sag (2000), comme en (20a,b) :

(20)a. A : Paul ga nakunat-ta. - B : Paul ga nakunat-ta-dat- te ? 
Paul Nom mourir-Pst -- Paul Nom mourir- Pst -Cop-vous dites ?

'A : Paul est mort. - B : Vous dites que Paul est mort ?'

b. A : Paul wa nihongo o eran-da. - B : Paul ga nihongo o eran- da(-dat)-te.

Paul Top japonais Acc choisir-Pst Paul Nom japonais Acc choisir-Pst-Cop-vous dites

'A : Paul a choisi le japonais. - B : Vous dites que Paul a choisi le japonais ?'

Ce parallélisme indique que le mot WH des questions de reprise (ex. qui en (15a) ou nani 'quoi' en $(19 \mathrm{a}, \mathrm{b})$ ) sert à mettre en relief (focaliser) la partie de la proposition citée qui est incompatible avec le fonds privé en arrière-plan du locuteur.

C'est, entre autres, Rooth (1996) qui caractérise le focus en termes de paire question-réponse. Son idée principale est que « la fonction essentielle du focus est d'évoquer l'ensemble de propositions contrastées (Alternative Set en anglais, et abrégé ALT ci-dessous) » et que « la relation entre les ALT et le focus peut être conçue comme une paire Question-Réponse ${ }^{14}$ (Rooth, 1996 : 276). Suivant la sémantique du monde possible où une proposition est définie comme un ensemble de mondes possibles $\mathrm{w}$, et une question comme un ensemble de propositions, le focus et ses ALT de (19bB) et (20bB) sont respectivement formalisés par (21a) et (21b) :

(21)a. $\quad \mathrm{p}=\lambda \mathrm{w}($ Paul a choisi le japonais en w) [focus : proposition citée]

b. $\quad \lambda \mathrm{q}[\mathrm{q}=\lambda \mathrm{w}(\exists \mathrm{x}($ Paul a choisi $x$ en $\mathrm{w}))]$ [ALT : ensemble de propositions formant le fonds privé]

c. $\quad \forall \mathrm{w}^{\prime} \in$ Fonds privé : $\mathrm{w}^{\prime} \notin \mathrm{p}=\lambda \mathrm{w}$ (Paul a choisi le japonais en $\mathrm{w}$ ) [condition d'appropriation]

Dans cette optique, les questions de reprise littérales ainsi que celles à mot WH mettent toutes les deux en oeuvre le focus. La différence est que les premières explicitent le focus lui-même, tandis que ce sont les ALT qui sont visibles dans les secondes. La condition d'appropriation des questions de reprise se réduit à dire que le focus (=à la proposition citée) ne fait pas partie des ALT (=ensemble de propositoins formant le fonds privé en arrière-plan du locuteur), comme le représente (21c).

\subsection{Questions de reprise et nante}

J'avancerai l'idée que la particule de focus nante en (22a,b), qui consiste dans le mot WH nan(i) ('quoi') et la marque de citation te, partage cette condition d'appropriation avec les questions de reprise :

(22)a. Paul ga nakunat-ta nante !

Paul Nom mourir-Pst NANTE

'Paul est mort, ce n'est pas possible / je ne peux pas le croire !'

b. Paul wa nihongo nante eran- da.

Paul Top japonais NANTE choisir-Pst

'Paul a choisi une langue comme le japonais (et non pas l'espagnol ou l'allemand) !'

D'autre part, tandis que les questions de reprise demandent à l'interlocuteur de confirmer la validité de la proposition citée dans le fonds partagé, l'étonnement exprimé par nante est essentiellement monologique, de même que les exclamatives en (10a,b,c). Beyssade \& Marandin (2006) définissent en effet l'exclamation comme non interactionnelle : à la différence des autres types de phrases (i.e. déclarative, interrogative et impérative), la phrase exclamative ne requiert que l'implication (commitemment) du locuteur et ne fait aucune demande à l'interlocuteur.

Ginzburg \& Sag (2000) associent en outre, avec Zanuttini \& Portner (2003), le type syntaxique Exclamative au type sémantique Fait ('Fact'). Le Fait est défini dans l'optique dialogique comme une proposition présupposée comme vraie dans le fonds partagé. Si on réduit le fait qu'une proposition se 
trouve dans le fonds partagé au fait qu'elle est vraie dans le monde actuel w0, les deux composantes de la sémantique de la particule nante (factivité et condition d'appropriation) sont représentées par (23a,b) :

(23) sémantique de nante (version à modifier)

a. $\quad \mathrm{w} 0 \in \mathrm{p}=\lambda \mathrm{w}$ (Paul a choisi le japonais en $\mathrm{w})$

[factivité]

b. $\forall \mathrm{w}^{\prime} \in$ Fonds privé : $\mathrm{w}^{\prime} \notin \mathrm{p}=\lambda \mathrm{w}$ (Paul a choisi le japonais en $\mathrm{w}$ ) [condition d'appropriation]

(23a) et (23b) correspondent respectivement à la factivité et à la condition d'élargissement en (13a,b), proposées par Zanuttini \& Portner (2003) pour les phrases exclamatives. Le fonds privé en arrière-plan en (23b) correspond au domaine initial D1 en (13b). La différence entre ma proposition et leur analyse est que la condition d'élargissement chez eux est présentée comme a priori, tandis que l'origine de la condition d'appropriation est explicitée comme une condition pragmatique imposée sur l'emploi approprié de la question de reprise. Il est à noter que ma proposition s'applique naturellement à l'adverbial exclamatif nante en (6a) qui consiste également en le mot WH nan et la marque de citation te.

\subsection{Nante et expressions modales}

Je tenterai ensuite de préciser la notion du fonds privé en arrière-plan en (23b), en me référant aux travaux antérieurs sur la modalité. Kadmon \& Landman (1993 : 381) soutiennent que «être surpris que A est toujours relatif à une certaine perspective en vertu de laquelle $A$ est surprenant. La perspective est un paramètre contextuellement déterminé pour l'interprétaion de être surpris, exactement de la même manière que la base modale ('modal base') est un paramètre contextuellement déterminé pour l'interprétation des auxiliaires modaux $»^{15}$.

La base modale est caractérisée par Kratzer (1981-2002) comme l'arrière-plan conversationnel par rapport auquel les expressions modales sont interprétées. La base modale est formellement définie comme une fonction prenant la paire du locuteur (noté dans ce qui suit par moi) et d'un monde possible w et rendant l'ensemble de mondes accessibles pour le locuteur à partir de ce monde possible. Cette fonction est signalée par $\mathrm{f}$ (moi, w). Par exemple, pour interpréter l'auxiliaire modal must en (24a), on ne tient normalement pas compte des mondes extravagants, comme celui où $\mathrm{Jackl}$ n'existe pas, ni celui où un martien a commis un meurtre, etc.. La base modale sert à exclure ces mondes contextuellement non pertinents :

(24)a. Jackl must have been the murderer. (Kratzer, 1991-2002: 639)

b. In view of the available evidence, Jackl must have been the murderer. (ibid.)

Les mondes accessibles sont par ailleurs ordonnés selon un critère. Ce critère est appelé source ordonnante ('ordering source') et est défini sémantiquement comme une fonction prenant la paire du locuteur (moi) et d'un monde possible w et rendant l'ensemble de propositions accessbibles. Cette fonction est signalée par g (moi, w). Par exemple, si (24a) est interprété comme (24b), l'expression in view of the available evidence fournit la source ordonnante épistémique. Les sources ordonnantes autres que de type épistémique sont stéréotypiques (en vertu du déroulement normal d'événements), déontiques (en vertu de la loi), boulétiques (en vertu de souhaits du locuteur), doxastiques (en vertu de croyances du locuteur), etc. La diversité des sources ordonnantes permet de rendre compte des divers effets modaux ${ }^{16}$.

Discutant la « perspective » pertinente, selon Kadmon \& Landman (1993), pour l'expression de l'étonnement, von Fintel (1999) la définit formellement comme une fonction de sélection choisissant l'ensemble des meilleurs mondes à partir des mondes accessibles (= la base modale $\mathrm{f}(\mathrm{mo}, \mathrm{w}, \mathrm{w})$ ) par rapport à la source ordonnante $g(m o i, w)$. Cette fonction de sélection est notée par $\max _{\mathrm{g}(m o i, \mathrm{w})} \mathrm{f}(m o i, \mathrm{w})$. Par exemple, en (25a), la source ordonnante est précisée comme doxastique par l'expression Given my high opinion on his moral character. En simplifiant l'analyse de von Fintel, on peut représenter la sémantique de be surprised en (25a) par (25b) et (25c): 
(25)a. Given my high opinion on his moral character, I was surprised that he stole the watch. (von Fintel, $1999: 113)$

b. $[[\text { surprised }]]^{\mathrm{f}, \mathrm{g}}(\mathrm{p})(m o i)(\mathrm{w})$ est défini ssi w0 $\in \mathrm{p}=\{\mathrm{w}$ : il a volé une montre en $\mathrm{w}\} \quad$ [factivité]

c. si défini, [[surprised $]]^{\mathrm{f}, \mathrm{g}}(\mathrm{p})($ moi $)(\mathrm{w})$ est vrai ssi $\forall \mathrm{w}^{\prime} \in \max _{\mathrm{g}(m o i, \mathrm{w})} \mathrm{f}(m o i, \mathrm{w}): \mathrm{w}^{\prime} \notin \mathrm{p}$

Dans cette optique, le fonds privé en arrière-plan en $(23 b)$ est reformulé par la fonction de sélection $\max _{\mathrm{g}(m o i, \mathrm{w})} \mathrm{f}(m o i, \mathrm{w})$, comme en $(26 \mathrm{~b})$ :

(26) sémantique de nante (version modifiée)

a. $\quad \mathrm{w} 0 \in \mathrm{p}=\lambda \mathrm{w}($ Paul a choisi le japonais en $\mathrm{w})$

[factivité]

b. $\quad \forall \mathrm{w}^{\prime} \in \max _{\mathrm{g}(m o i, \mathrm{w})} \mathrm{f}(m o i, \mathrm{w}): \mathrm{w}^{\prime} \notin \mathrm{p}=\lambda \mathrm{w}($ Paul a choisi le japonais en $\mathrm{w})$

[condition d'appropriation]

La diversité des valeurs affectives de nante peut être saisie au moyen des différents types de source ordonnante, comme épistémique (en vertu des faits, ex. " pour Paul qui est francais, le japonais est une langue difficile à apprendre »), doxastique (en vertu de croyances du locuteur, ex. « le locuteur croyait que Paul s'intéressait à l'italien »), etc ${ }^{17}$.

Il en est de même pour les cas où nante porte sur la phrase entière comme en (27), dont la sémantique est représentée par $(28 \mathrm{a}, \mathrm{b})$ :

$$
\text { Paul ga nakunat-ta nante ! }
$$

Paul Nom mourir-Pst NANTE

'Paul est mort, ce n'est pas possible / je ne peux pas le croire !'

(28)a. $\quad \mathrm{w} 0 \in \mathrm{p}=\lambda \mathrm{w}($ Paul est mort en $\mathrm{w})$

[factivité]

b. $\quad \forall \mathrm{w}^{\prime} \in \max _{\mathrm{g}(m o i, \mathrm{w})} \mathrm{f}(m o i, \mathrm{w}): \mathrm{w}^{\prime} \notin \mathrm{p}=\lambda \mathrm{w}($ Paul est mort en $\mathrm{w}) \quad$ [condition d'appropriation]

\subsection{Valeur affective de nante comme implicature conventionnelle}

Je proposerai enfin d'analyser les valeurs affectives de nante (due à sa condition d'appropriation) comme des implicatures conventionnelles au sens de Potts (2005) et Kratzer (2005) ${ }^{18}$. Selon ces auteurs, l'implicature conventionnelle, transmise par les items expressifs comme damn en (29), ne contribue pas aux conditions de vérité, mais ne concerne que les conditions d'appropriation ${ }^{19}$ :

We have to look after Sheila's damn dog. (Potts, 2005 : 166)

\subsubsection{Symptômes de l'implicature conventionnelle}

Potts (2005) fait remarquer au moins quatre symptômes des items expressifs manifestant une implicature conventionnelle.

(I) L'item expressif adjectival comme damn en (29) peut évoquer un décalage entre la structure de surface et le contenu : l'évaluation peut porter pas seulement sur le NP modifié mais sur la phrase entière. Ainsi le dégoût exprimé par damn en (29) n'est pas nécessairement dû au chien de Sheila, (ex. il se peut que le locuteur l'aime bien dans une autre situation), mais au fait que le locuteur doit s'en occuper.

(II) L’évaluation n'est pas présupposée, mais impliquée : la présupposition évoquée par stop, «John fumait », peut être annulée en (30a), tandis que le dégoût évoqué par bastard n'est pas annulé en (30b) :

(30)a. John stopped smoking. But in fact, he has never smoked.

b. That bastard Corner was promoted. \# But probably he in fact is a nice guy. (idem.157) 
(III) L'évaluation est indépendante du contenu vériconditionnel. Elle peut être contredite sans nier celuici, comme en (31a), tandis qu'elle n'est pas influencée par la négation de la phrase, comme en (31b) :

(31)a. Anne: That bastard Corner was promoted. - Kyle: Yes, but Corner is not a bastard. (ibid.)

b. It is just not true that Sheila's damn dog is on the couch! (idem. 159)

(IV) L'évaluation reflète en principe exclusivement la perspective du locuteur : le dégoût exprimé par damn en (32) n'est pas attribué au sujet de la phrase, Clinton, mais seulement au locuteur, Bush. (32) peut ainsi être suivi par « mais Clinton n'a jamais dit que les républicains sont dégoûtants », mais non par " mais je ne suis pas sûr que les républicains sont dégoûtants » :

$$
\text { Bush : Clinton says the damn Republicans should be less partisan. (idem.160) }
$$

\subsubsection{Comportements de nante}

La particule nante manifeste de fait ces quatre symptômes.

(I) La valeur affective de nante peut porter sur la partie décalée de la structure de surface. En (33), il se peut que le locuteur apprécie le japonais (superficiellement focalisé) comme une langue intéressante, tandis qu'il trouve étonnant le fait que Paul ait choisi le japonais comme langue étrangère :

(33) Paul wa nihongo nante eran- da.

Paul Top japonais NANTE choisir-Pst

'Paul a choisi une langue comme le japonais (et non pas l'espagnol ou l'allemand) !'

(II) La valeur affective de nante n'est pas annulable, comme en (34) :

(34) Paul wa nihongo nante eran- da. \#Demo zituwa sore wa toozenda.

Paul Top japonais NANTE choisir-Pst mais en fait cela Top natural

'Paul a choisi une langue comme le japonais ! Mais cela est naturel.'

(III) L'évaluation de nante peut être contredite sans nier le contenu vériconditionnel, comme en (35a). D'autre part, la négation de la phrase n'influence pas le jugement du locuteur (i.e. le choix du japonais par Paul comme langue étrangère est étonnant), en (35b) :

(35)a. A : Paul wa nihongo nante eran- da. - B : aa, demo toozenja-nai-ka

Paul Top japonais NANTE choisir-Pst oui, mais naturel-Neg-Interrogatif

'A : Paul a choisi une langue comme le japonais ! - B : Oui, c'est vrai. Mais ce choix est naturel, n'est-ce pas?'

b. Paul wa nihongo nante eraba-nakat-ta.

Paul Top japonais NANTE choisir-Neg-Pst

'Paul n'a pas choisi une langue comme le japonais !'

(IV) La valeur affective de nante ne peut être attribuée qu'au locuteur, comme en (36) :

(36) Paul ga nihongo nante eran- da koto ni Jean wa odoroi- ta. \#demo watasi wa

Paul Nom japonais NANTE choisir-Pst fait Loc Jean Top s'étonner-Pst mais moi Top

odoroka-nakat-ta.

s'étonner-Neg-Pst

'Le fait que Paul ait choisi une langue comme le japonais a étonné Jean, mais cela ne m'a pas étonné. 
Ces points communs justifient l'analyse des valeurs affectives de nante comme des implicatures conventionnelles.

\section{Phrases nominales événementielles du français}

Sur la base des résultats de la section 3, cette section envisagera les phrases nominales événementielles du français. J'avancerai d'abord des hypothèses sur leur syntaxe et leur sémantique ( $\$ 4.1$ ). Puis, je présenterai certains arguments en faveur de ces hypothèses ( $\S 4.2$ ). Je montrerai enfin que l'analyse proposée pour nante dans la section 3.4 rend également compte de la diversité des valeurs affectives transmises par les phrases nominales événementielles, aussi bien que d'un point commun entre cette construction et le conjonctif concessif quoique (§ 4.3).

\subsection{Hypothèses}

\subsubsection{Sémantique}

Je soutiens d'abord que la phrase nominale en (37) est associée, comme la particule de focus nante, aux questions de reprise en $(38 \mathrm{a}, \mathrm{b})$ exprimant un étonnement : le locuteur B n'accepte pas tout de suite la vérité de la propositon que l'interlocuteur A vient d'énoncer, " Paul est mort ». Autrement dit, ces questions exigent que la proposition citée ne soit pas vraie dans son fonds privé en arrière-plan (condition d'appropriation) :

(37) Paul qui est mort!

(38)a. A : Paul est mort. - B : Qui est mort?

b. A : Paul est mort. - B : Quoi ?

Toutefois, tandis que les questions de reprise demandent à l'interlocuteur de confirmer la validité de la proposition citée dans le fonds partagé, les phrases nominales comme (37), de même que les phrases exclamatives et les phrases incluant la particule nante, ne sont pas interactionnelles et présupposent que la proposition citée est vraie dans le fonds partagé (factivité). La sémantique de (37) est donc représentée $\operatorname{par}(39 \mathrm{a}, \mathrm{b})$ :

sémantique de la phrase nominale événementielle

$$
\begin{aligned}
& \text { a. } \quad \mathrm{w} 0 \in \mathrm{p}=\lambda \mathrm{w}(\text { Paul est mort en } \mathrm{w}) \\
& \text { [factivité] } \\
& \text { b. } \left.\forall \mathrm{w}^{\prime} \in \max _{\mathrm{g}(m o i, \mathrm{w})} \mathrm{f}(m o i, \mathrm{w}): \mathrm{w}^{\prime} \notin \mathrm{p}=\lambda \mathrm{w} \text { (Paul est mort en } \mathrm{w}\right) \quad \text { [condition d'appropriation] }
\end{aligned}
$$

Je soutiens aussi que la condition d'appropriation en (39b) est, comme dans le cas de la particule de focus nante, une implicature conventionnelle.

\subsubsection{Syntaxe}

Je suppose par ailleurs avec Sasse (1987), Léard (1992), Lambrecht (1994), Furukawa (1996), entre autres, que cette construction met en relief la phrase entière, en créant un décalage entre la structure de surface et la sémantique. Autrement dit, elle place le focus sur la phrase (i.e. jugement thétique). La syntaxe de (37) est ainsi représentée par (40), dont l'opérateur WH est mis en oeuvre syntaxiquement par la construction focalisante :

$$
\begin{aligned}
& \text { syntaxe de la phrase nominale événementielle (37) } \\
& \left.\left.\left[\text { FocP Paul qui est mort [Foc (Foc) [CP WH Op }{ }_{k}\left[\mathrm{C} \text { qui }\left[\mathrm{IP}_{\mathrm{k}}\right]\right]\right]\right]\right]
\end{aligned}
$$

Cette hypothèse est inspirée par l'analyse de Doetjes, Rebuschi \& Rialland (2004) qui paraphrasent (41aB) par (41b), dont la partie soulignée est implicite en (41aB) : 
(41)a. A : Tu sembles inquiète. Qu'est-ce qui se passe ?

B : C' est le petit qui est tombé dans l'escalier. (Clech-Darbon, Rebuschi \& Rialland, 1999 : 84)

b. = C'est [le petit qui est tombé dans l'escalier] [Cp qui me tracasse]. (Doetjes, Rebuschi \& Rialland, $2004: 540)$

De même que le CP souligné en (41b) introduit les ALT, le CP phonétiquement nul en (40) donne lieu aux ALT incluant la variable d'événement Ev en (42b) et correspondant à la question de reprise quoi? en (38b) :

$$
\begin{aligned}
\text { (42)a. } & \mathrm{p}=\lambda \mathrm{w}(\text { Paul est mort } \mathrm{en} \mathrm{w}) \\
\text { b. } & \lambda \mathrm{q}[\mathrm{q}=\lambda \mathrm{w}(\exists \mathrm{Ev}(\text { Ev se produit } \mathrm{en} \mathrm{w}))]
\end{aligned}
$$$$
\text { [focus] }
$$

Du point de vue compositionnel, (37) n'engendre que le focus en (42a) et les ALT en (42b). Il est à noter que le focus correspond à la proposition citée qui est vraie dans le fonds partagé, tandis que les ALT correspondent à l'ensemble des propositions qui sont vraies dans le fonds privé en arrière-plan du locuteur. Dans cette optique, l'absence de la principale dans les phrases nominales est motivée pour indiquer l'absence de la mise en relation entre le focus et les ALT, voire leur incompatibilité. D'autre part, les proposition principales comme il y a / c'est dans les constructions il y a NP qui VP / c'est NP qui $V P$, servent à préciser la relation entre le focus et les ALT comme existentielle (ou additive) ou comme identificationnelle (ou exclusive).

Cette hypothèse selon laquelle l'absence de la principale a une motivation pragmatique s'accorde avec la remarque de Le Bidois \& Le Bidois (1971 : 379) selon laquelle «Ces conjonctives [=les phrases nominales comme (37)] sont des principales sans aucune dépendance avec une autre proposition » et avec celle de Sandfeld (1965: 154-156) qui soutient que «Ici, rien n'est omis ».

\subsection{Arguments}

Certains arguments viennent à l'appui de ces hypothèses.

\subsubsection{En faveur de la nature événementielle}

D'abord, la nature événementielle des phrases nominales est confirmée par les trois tests suivants :

(I) De même que les phrases nominales exclamatives comme (10c), les phrases nominales de type (37) sont reprises par un pronom neutre qui ne manifeste aucun accord de genre et de nombre, même lorsque le NP focalisé dénote clairement une entité et non pas un événement, comme deux jurys en $(43)^{20}$ :

(43) Deux jurys qui condamnent un homme, ça vous impressionne. (Furukawa, $1996: 49$ )

(II) La marque plurielle attachée au NP est légitimée par sa dépendance par rapport au VP dans la séquence NP qui VP (pluralité dépendante). Ainsi, (44) ne permet que la lecture paraphrasée par « il se produit sans arrêt des événements où au moins un soldat meurt». Ceci indique que le NP les soldats est sémantiquement intégré au prédicat mourir, dont l'ensemble est interprété sous la portée de l'opérateur itératif introduit par sans arrêt :

$$
\text { [Le Président a déclaré que la guerre était finie en Irak] }
$$

Et les soldats qui meurent sans arrêt !

(III) Cette construction ne peut être enchâssée que sous les verbes principaux qui prennent un objet événementiel, comme les verbes de perception, les verbes existentiels (ex. il y $a, j$ 'ai), ou les présentatifs (c'est, voilà). Si la séquence $N P$ qui $V P$ se trouve après les verbes qui prennent un objet d'entité comme rencontrer, attraper, etc., comme en (45a), la subordonnée qui VP est interprétée comme un prédicat second adjoint au VP principal, comme en (45b) : 
(45)a. Elle l'aperçut qui se peignait soigneusement. (Saint-Exupéry)
b. [vp [vp l'apercevoir][CP qui se peignait]]
(à la Cinque 1995)

\subsubsection{En faveur de l'hypothèse du focus}

L'hypothèse que cette construction met en oeuvre un focus est confirmée par les deux arguments suivants

(I) Une valeur affective analogue est exprimée en anglais par les constructions dites superlatives quantificationnelles ('quantificational superlative') incluant un focus contrastif, comme en (46) :

(46) John would distrust Albert SCHWEITzer. (Krifka, $1995: 15)$

Selon Krifka (1995 : 15), « la fonction du focus emphatique est d'indiquer que la proposition affirmée est particulièrement inappropriée aux ALT, étant donné les connaissances partagées. [(46)] suggère que John devrait avoir plus de confiance en Albert Schweitzer qu'en toute autre personne $»^{21}$.

Bien que les superlatives quantificationnelles évoquent, selon Krifka, les ALT décrivant l'ensemble des propositions qui sont vraies dans le fonds partagé, tandis que les phrases nominales du français introduisent les ALT correspondant à l'ensemble des propositions qui sont vraies dans le fonds privée du locuteur, les deux constructions ont un point commun : elles expriment l'étonnement au moyen de l'incompatibilité entre le focus et les ALT.

(II) La construction à focus emphatique en anglais peut par ailleurs exprimer le focus sur la phrase. Lambrecht (1994) met en évidence que le focus sur la phrase n'est possible que lorsque le sujet est accentué, comme l'indique le contraste entre (47a) et (47b), où le contenu de la question appelle une réponse focalisant la phrase entière :

(47)a. A : What happened? - B : Her FATHER died. (Lambrecht, 1994 : 143)

b. A : Why didn't she come to work today? - B : \# Her husband made a SCENE. (idem.308)

Léard (1992 : 131) observe que les phrases nominales du français « valent seulement pour le sujet, ou dit autrement, sont toujours introduites par qui ». L'asymétrie sujet-objet est confirmée par le contraste entre (48a) et (48b) :

(48)a. Tiens ! Le facteur qui passe! (Furukawa, $1996: 64$ )

b. ?Oh! Le facteur que Pierre renverse ! (ibid.) $)^{22}$

Cette contrainte commune justifie la mise en parallèle de la construction à focus emphatique de l'anglais et des phrases nominales du français.

\subsubsection{En faveur de la nature exclamative}

Comme on l'a vu dans $\S 2.3$, la nature exclamative des phrases nominales de type Les beaux biceps que tu as! est confirmée par la factivité, qui est discernée dans l'incompatibilité avec la négation ou l'interrogation de la principale. Kleiber (1988) fait effectivement remarquer que la principale dominant une phrase nominale ne peut être négative, ni interrogative, ni contruites avec les auxiliaires modaux qui risquent d'influencer la vérité de la proposition subordonnée, comme en (49a,b,c) :

(49)a. *Paul n'a pas vu Marie qui pleurait. (adapté de Kleiber 1988)

b. *Est-ce que Paul a vu Marie qui pleurait? (ibid.)

c. *Paul doit / peut / veut / croit voir Marie qui pleure. (ibid.) 


\subsubsection{En faveur de l'hypothèse des implicatures conventionnelles}

Les phrases nominales événementielle du français manifestent, de même que la particule nante du japonais, les symptômes de l'implicature conventionnelle.

(I) Le décalage entre la syntaxe de surface et la portée de la valeur affective est claire. Bien que (37) ne focalise en surface que le NP Paul, la valeur affective porte sur l'événement dénoté par la phrase entière, à savoir la mort de Paul.

(II) La valeur affective n'est pas annulable comme en (50) :

(50) Paul qui est mort! \# Mais cela est en fait naturel.

(III) La valeur affective peut être contredite par l'interlocuteur sans nier le contenu véri-conditionnel en (51), ce qui indique l'indépendance de la valeur affective par rapport au contenu véri-conditionnel :

(51) A : Paul qui est mort ! - B : Qui, c'est vrai. Mais ce n'est pas étonnant. Il buvait trop.

(IV) La valeur affective ne peut être attribuée qu'au locuteur, comme en (52) :

(52) *Paul qui est mort, cela est étonnant pour Jean. Mais cela ne m'a pas étonné.

Ces points communs semblent appuyer l'analyse de la valeur affective des phrases nominales événementielles en termes d'implicature conventionnelle ${ }^{23}$.

\subsection{Avantages des hypothèses avancées}

Cette section présentera certains avantages des hypothèses syntaxiques et sémantiques avancées en $\S 4.1$.

\subsubsection{Diverses valeurs affectives}

L'hypothèse sémantique $(39 \mathrm{a}, \mathrm{b})$, reprise en $(54 \mathrm{a}, \mathrm{b})$, permet de rendre compte des diverses valeurs affectives attribuées aux phrases nominales (ex. étonnement, désaccord, regret, etc.) grâce à la diversité de la source ordonannte contextuellement spécifiée g $(m o i, w)$, sans devoir supposer, comme Michaelis \& Lambrecht (1996), des prédicats affectifs différents pour les différents cas. Par exemple, s'il s'agit de la source ordonnante épistémique (ex. si le locuteur a récemment rencontré un Paul très en forme) ou stéréotypique (ex. si Paul est encore jeune), (53) exprime un certain étonnement. Avec un type doxastique (ex. si le locuteur croyait que Paul était très en forme), on sent un désaccord. Si l'on a affaire au type boulétique (ex. si le locuteur espérait que Paul vivrait longtemps), on a une valeur de regret. La condition d'appropriation en (54b) est intuitivement schématisée en (54c) :

(53) Paul qui est mort!

(54)a. $\quad \mathrm{w} 0 \in \mathrm{p}=\lambda \mathrm{w}$ (Paul est mort en $\mathrm{w}) \quad(=(39 \mathrm{a})$

[factivité]

b. $\quad \forall \mathrm{w}^{\prime} \in \max _{\mathrm{g}(m o i, \mathrm{w})} \mathrm{f}(m o i, \mathrm{w}): \mathrm{w}^{\prime} \notin \mathrm{p}=\lambda \mathrm{w}($ Paul est mort en $\mathrm{w})(=(39 \mathrm{~b})$ [condition d'appropriation]

c. focus [=proposition citée « Paul est mort »] $\notin$ ALT [= ensemble de propositions consistant en mondes accessibles maximalement proches des faits disponibles (épistémique), du déroulement naturel des événements (stéréotypique), des croyances du locuteurs (doxastique), ou de ses souhaits (boulétique)]

\subsubsection{Contraste avec le discours précédent}

Les cas où les phrases nominales expriment un contraste avec le discours précédent comme en (1b), repris en (55a), peuvent être analysés comme des emplois élargis : le focus ne correspond plus à la proposition citée, mais à la proposition que le locuteur énonce lui-même ; les ALT ne correspondent plus à l'ensembles des propositions vraies dans le fonds privé, mais à celui des propositions inférées au moyen 
des connaissances générales, à partir de la proposition que le locuteur vient d'énoncer lui-même. En bref, les cas du contraste avec le discours précédent impliquent, comme aux cas exprimant les valeurs affectives, que le focus ne fait pas partie des ALT. Mais les contenus du focus et des ALT sont différents dans les deux cas, comme en (55b) :

(55)a. Nous sommes à la gare, déjà ! Moi qui avais encore tant de choses à vous conter. (=(1b))

b. focus [proposition que le locuteur énonce $=« j$ 'avais encore tant de chose à vous conter $»]$ ALT [ = ensemble des propositions inférées du fait que " nous sommes à la gare », ex. les interlocuteurs s'étaient suffisamment parlé, ils devront se séparer bientôt, etc.)]

\subsubsection{Traitement analogue du conjonctif concessif quoique}

Dans cette optique, il est possible de rapprocher l'opérateur WH implicite invoqué selon l'hypothèse syntaxique en (40), dans la phrase nominale (55a), de quoi inclus dans le conjonctif de concession quoique en (56a). Morel (1996:26) donne en effet une définition de quoique analogue à la sémantique décrite en (55b). Selon cet auteur, le conjonctif quoique "marque une opération de parcours sur les propriétés différentielles associables au prédicat de la principale » : quoique en (56a) sert ainsi à parcourir l'ensemble des propositions associables à la proposition principale « Jean part », par exemple «Il fait beau », etc. Le sens concessif se réduit au fait que la proposition décrite par la subordonnée « Il pleut » ne fait pas partie de l'ensemble des propositions associables, comme en (56b) :

(56)a. Quoiqu'il pleuve, Jean part. (=(2))

b. focus [proposition subordonnée=« il pleut $»] \notin$ ALT $[=$ ensemble de propositions inférées de la proposition principale « Jean part »]

L'hypothèse avancée permet ainsi de saisir le point commun entre le contraste exprimé par les phrases nominales événementielles de type (55a) et le sens concessif exprimé par le conjonctif quoique comme en (56b), au moyen de l'existence de l'opérateur WH qui est soit visible (pour quoique), soit implicite (pour les phrases nominales).

\section{Conclusion}

Les résultats de cette étude peuvent être récapitulés comme suit.

(I) De même que les phrases nominales exclamatives (ex. "The things that he ate! »), les phrases nominales événementielles (ex. "Paul qui est mort! ») ne sont pas des phrases elliptiques dont le thème ou le rhème implicites sont contextuellement récupérés, mais des phrases indépendantes dont l'ensemble est focalisé. La sémantique du focus a deux composantes, focus et ALT.

(II) De même que la particule de focus nante, les phrases nominales événementielles partagent, avec les questions de reprise, la condition d'approciation selon laquelle le focus (correspondant à la proposition citée) ne fait pas parties des ALT (qui consite en ensemble des propositions vraies dans le fonds privé en arrière-plan du locuteur). La condition d'appropriation apporte des valeurs affectives qui doivent être analysées comme des implicatures conventionnelles. Cette construction est de plus non interactionnelle : elle présuppose que la proposition citée dénote un Fait, c'est-à-dire, qu'elle est vraie dans le fonds partagé (factivité).

(III) Les ALT invoqués par les phrases nominales sont contextuellement restreints, comme dans les cas des auxiliaires modaux. Cette restriction contextuelle permet de rendre compte du fait que cette construction apporte diverses valeurs affectives (ex. étonnement, regret, désaccord, contraste, etc.) selon le contexte, de même que les auxiliaires modaux expriment diverses types de modalité (ex. épistémique, boulétique, doxastique, stéléotypique, etc.) en fonction du context.

(IV) L'hypothèse que cette construction met en oeuvre un opérateur WH implicite permet de saisir un point commun entre le cas du contraste (ex. "Nous sommes à la gare! Moi qui avais encore tant de 
choses à vous conter ») et le conjonctif concessif quoique (ex. "Quoiqu'il pleuve, Jean part ») qui contient visiblement le mot WH quoi.

\section{Références bibliographiques}

Beyssade, C. \& Marandin, J.-M. (2006). The Speech Act Assignment Problem Revisited: Disentangling Speaker's Commitment from Speaker's Call on Adressee. In Bonami, O. \& Cabredo Hofherr, P. (éd.), Empirical Issues in Syntax and Semantics $6: \mathrm{http}: / / w w w . c s s p . c n r s . f r / e i s s 6,37-68$.

Cinque, G. (1995). Italian Syntax and Universal Grammar. Cambridge : Cambridge Univ. Press.

Clech-Darbon A., Rebuschi G., Rialland A. (1999). Are there Cleft Sentences in French? In Tuller, L. \& Rebuschi, G. (éd.), The Grammar of Focus. Amsterdam : Benjamins, 83-118.

Doetjes, J., Rebuschi, G. \& Rialland, A. (2004). Cleft Sentences. In Corblin, F. \& de Swart, H. (éd.), Handbook of French Semantics. Stanford : CSLI, 529-552.

Erteschik-Shir, N. (1997). The Dynamics of Focus Structure. Cambridge : Cambridge Univ. Press.

Furukawa, N. (1996). Grammaire de la prédication seconde. Louvain-la-Neuve : Duculot.

Guasti, M.-T. (1992). Causative and Perception Verbs. Thèse de doctorat. Univ. de Genève.

Jäger, G. (2001). Topic-Comment Structure and the Contrast between Stage Level and Individual Level Predicates. Journal of Semantics 18, 83-126.

Kleiber, G. (1988). Sur les relatives de type Je le vois qui arrive. Travaux de Linguistique, 17, 89-115.

Kratzer, A. (1981-2002). The Notional Category of Modality. In Portner, P. \& Partee, B. (éd.), Formal Semantics: The Essential Readings, Oxford : Blackwell, 289-323.

Kratzer, A. (2005). Interpreting focus: presupposed or expressive meaning? A comment on Geurts \& van der Sandt. Theoretical Linguistics, à paraître. disponible sur le site SemanticArchive.

Krifka, M. (1995). The Semantics and Pragmatics of Polarity Items. Linguistic Analysis, 25, 1-49.

Lambrecht, K. (1994). Information Structure and Sentence Forms. Cambridge : Cambridge Univ. Press.

Léard, J.-M. (1992). Les gallicismes. Louvain-la-Neuve : Duculot.

Le Bidois, G. \& Le Bidois, R. (1971). Syntaxe du français moderne, ses fondements histriques et psychologiques, tome II. Paris : Picard.

Lefeuvre, F. (1999). La phrase averbale en français. Paris / Montréal : L’Harmattan.

Marandin, J.-M. (2005), Formatage de l'information : focus et contexte, In Corblin, F. \& Gardent, C. Interpréter en contexte. Paris : Lavoisier.

Michaelis, L. \& Lambrecht, K. (1996). Toward a Construction-Based Theory of Language Function. Language 72.2, 215-247.

Morel, M.-A. (1996). La concession en français. Paris : Ophrys.

Nihon Kokugo Daijiten (Le Grand dictionnaire de la langue japanaise). (2003). vol.10, seconde édition. Tokyo : Shogakukan Pub.

Numata, Y. (2000). Toritate (Particles de focus), in Kinsui, S., Kudo, M. \& Numata, Y. (éd.), Toki, hitee to toritate (Temps, négation et particules de focus), Tokyo : Iwanami Pub, 153-216.

Portner, P. \& Zanuttini, R. (2005). The Semantics of Nominal Exclamatives. In Elugardo, R. \& Stainton, R.-J. (éd.), Ellipsis and Nonsentential Speech. Amsterdam : Springer, 57-67.

Potts, C. (2005). The Logic of Conventional Implicatures. Oxford : Oxford University Press.

Rooth, M. (1996). Focus, In Lappin, S. (éd.), The Handbook of Contemporary Semantic Theory, Oxford : Blackwell, 271-297.

Sandfeld, K. (1965). Syntaxe du français contemporain 2 : Les propositions subordonnées, Genève : Droz. 
Sasse, H.-J. (1987). The Thetic / Categorical Distinction Revisited, Linguistics, 25, 511-580.

Teramura, H. (1991). Nihongo no sintakusu to imi III (Syntaxe et sémantique du japonais III), Tokyo : Kurosio Pub.

von Fintel, K (1999). NPI-licensing, Strawson-entailment and context-dependency. Journal of Semantics, 16.2, 97148.

Yamanaka, M. (2007). Gendai Nihongo ni okeru Toritate-Josi no Kenkyuu (Une étude sur les particules de focus en japonais contemporain), Tokyo : Kurosio Pub.

Zanuttini, R. \& Portner, P. (2003). Exclamative Clauses: at the Syntax-Semantics Interface, Language, 79.1, 39-81.

\footnotetext{
${ }^{1}$ Je remercie Anne Zribi-Hertz et les deux rapporteurs qui ont revu le manuscrit de cette étude pour leurs remarques précieuses et constructives Je suis reconnaissant à Danièle Godard d'avoir attiré mon attention sur Marandin (2005). Merci aussi à mon collègue d'Okayama, Michel de Boissieu, pour la correction du français. Toutes les insuffisances qui restent sont dues à l'auteur. Cette étude a reçu le soutien financier de la Japan Society for the Promotion of Sciences (numéro 19520346).

${ }^{2}$ Un des rapporteurs fait remarquer que les énoncés qui ne consistent qu'en un verbe infinitif, comme en (I), transmettent également une valeur affective :
}

(I) Et puis il y avait mes tantes. Bavarder ! Et manger des chocolats !

Je ne sais pour le moment si ces phrases doivent être traitées parallèlement avec les phrases nominales. Ce sera le sujet d'une étude ultérieure.

${ }^{3}$ Les abréviations utilisées dans cette étude sont les suivantes : Acc $=$ accusatif $;$ Comp $=$ complémenteur ; Cop = copule ; $\mathrm{CP}=$ syntagme de complémenteur $; \mathrm{DP}=$ syntagme de déterminant $; \mathrm{Gen}=$ génitif $;$ Loc = locatif $;$ Nom = nominatif $; \mathrm{Op}=$ opérateur $; \mathrm{Neg}=$ négation $; \mathrm{NP}=$ syntagme nominal $; \mathrm{Pst}=$ passé $;$ Top $=$ topique $; \mathrm{VP}=$ syntagme verbal ; \#= bien formé syntaxiquement, mais inapproprié dans son contexte discursif.

${ }^{4}$ Plus exactement, la remarque de Numata (2000) porte sur une autre particule de focus, nanka, qui transmet une valeur affective analogue à nante.

${ }^{5}$ La présente étude ne peut pas traiter de la fonction de la copule $d a$ dans les questions de reprise, qui sera le sujet d'une étude ultérieure.

${ }^{6}$ En fait, diachroniquement, la source immédiate de la particule de focus nante n'est pas la même que celle de l'adverbe exclamatif nante. Selon le Nihon Kokudo Daijiten (2003:341), la première est dérivée de nado to 'quelque chose de ce genre + citation', tandis que la seconde vient de nani to iu 'quoi + citation + dire'. Il est toutefois à noter que nado est dérivé de nani-to 'quoi + citation'. Du coup, les deux nante incluent étymologiquement le mot WH, nani 'quoi', et le complémenteur de citation.

${ }^{7}$ Leurs analyses sont illutrées par les citations suivantes : « the distinction between sentences with stage topics [i.e. spatio-temporel topic] and sentences with individual topics is equivalent to the thetic-categorical distinction " (Erteschik-Shir, 1997 : 241) ; "thetic clauses are not really topic-less but [..] the reported event is the topic [...] Clauses without an overt topic are always thetic, i.e. construe the event argument of the predicate as topic » (Jäger, $2001: 114$ )

${ }^{8}$ « an elliptical construction in which the affective judgment is pragmatically inferred ».

${ }^{9}$ " the WH phrase binds a variable for which an appropriate value cannot be found in the contextually given domain » (Zanuttini \& Portner, $2003: 50$ ).

${ }^{10}$ Plus exactement, la condition d'élargissement est définie par (I) (Zanuttini \& Portner, $2003: 52$ ) :

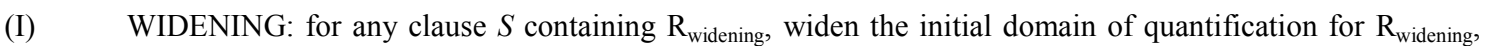
D1, to a new domain, D2, such that

a. $\quad[[S]] \mathrm{w}, \mathrm{D} 2,<-[[S]] \mathrm{w}, \mathrm{D} 1,<\neq 0$ and

b. $\forall \mathrm{x} \forall \mathrm{y}[\mathrm{x} \in \mathrm{D} 1 \& \mathrm{y} \in(\mathrm{D} 2-\mathrm{D} 1) \rightarrow \mathrm{x}<\mathrm{y}]$ 


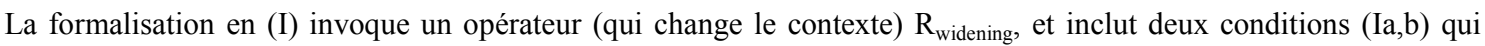
expriment conjointement l'extrémité. Pour la phrase exclamative What things he eats!, «[[S]]w,D2,< is a set of true (in w) propositions of the form 'he eats $\mathrm{x}$ ' where $\mathrm{x}$ is drawn from the new domain $\mathrm{D} 2$, while $[[S]] \mathrm{w}, \mathrm{D} 1,<$ is the corresponding set for the initial domain D1. [(Ia)] amounts to requiring that new things that he eats $[=$ some surprising food, like very spicy habaneros] be added to the domain. And (Ib) says that for any member $\mathrm{x}$ among the initial domain D1 and for any member y among the widened part D2-D1, y is higher than $\mathrm{x}$ on the relevant scale » (Zanuttini \& Portner, 2003 : 52).

${ }^{11}$ La syntaxe en (14) est conçue dans le cadre de l'antisymétrie : le DP prend, comme son complément, le CP.

${ }^{12}$ Le texte original est le suivant :

(I)a. Tracy: You like WHO ? (Ginzburg \& Sag, $2000: 260$ )

b. Tracy was amazed at what I told her, so she asked me who it was that I have said I like. (ibid.)

${ }^{13}$ La présentation des analyses de Ginzburg (à paraître) dans cette étude est largement due à celle effectuée dans Marandin (2005).

${ }^{14}$ « evoking alternatives is the general function of focus [and the relation between the alternatives and the focus] can be illustrated with the question-answer paradigm $»$.

${ }^{15}$ «To be surprised that $A$ is always relative to a certain perspective on A, a perspective that determines what is about $\mathrm{A}$ that is surprising and in virtue of what it is surprising. The perspective is a contextually determined parameter in the interpretation of surprised, very much in the same way that a 'modal base' is a contextually determined parameter in the interpretation of modals $»$.

${ }^{16}$ Chaque auxiliaire modal est en général lexicalement spécifié pour certains types de sources ordonnantes : pouvoir et devoir sont ainsi spécifiés pour les types épistémique et déontique.

${ }^{17}$ Le point commun entre la particule de focus nante et les expressions modales est également remarqué par Teramura (1991 : 188) (plus exactement, la remarque de Teramura (1991) porte sur une autre particule de focus nado, qui transmet une valeur affective analogue). Cet auteur recourt à une dichotomie entre le dictum concernant la relation prédicative entre le prédicat verbal et les arguments (ou les adjoints) et le modus décrivant une évaluation subjective du locuteur, et suppose que le modus est superposé au dictum. Il soutient ensuite que la particule nante est ajoutée, comme les expressions modales (exprimant le modus), après que la relation prédicative (correspondant au dictum) a été encodée. Cette analyse est appuyée par le fait que la particule nante suit nécessairement la marque casuelle comme en (Ia), et ne s'intercale jamais entre le NP et la marque casuelle, comme le montre (Ib):

(I)a. Paul wa France ni nante ika-nakat-ta.

Paul Top France Loc NANTE aller-Neg-Pst

'Paul n'est pas allé dans un pays comme la France'

b. *Paul wa France nante ni ika-nakat-ta.

Paul Top France NANTE Loc aller-Neg-Pst

${ }^{18}$ L'analyse en terms des implicatures conventionnelles a été proposée par un des rapporteurs.

${ }^{19}$ " the expressive meaning [...] would be the set of possible situations in which [the truth conditional content] is uttered appropriately » (Kratzer, $2005: 8$ )

${ }^{20}$ Comme le fait remarquer un des rapporteurs, un NP peut être repris par un pronom neutre sans la relative lorsqu'il dénote un événement comme en (I) :

(I) Des catastrophes comme celles-là, ça vous impressionne.

21 " The function of emphatic focus is to indicate that the proposition that is actually asserted is prima facie a particularly unlike one with respect to the alternatives [...] in the current common ground: $[\ldots]$ it must be considered that John would trust Albert Schweitzer than [...] any other person ».

${ }^{22}$ Léard (1992 : 131) attribue cette asymétrie à la faible topicalité du NP focalisé : en supposant que les phrases nominales événementielles apportent un jugement thétique dont tous les éléments apportent une information 
nouvelle, cet auteur soutient qu' « en l'absence de hiérarchie dans l'information apportée par les éléments, tous nouveaux, il n'y a pas de raison de bouleverser l'ordre des constituants ». Un des rapporteurs fait toutefois remarquer un contre-exemple (Ia) où l'objet est mis en relief. Cet exemple n'est pas un cas exceptionnel, comme le confirme l'existence d'exemples attestés comme (Ib) :

(I)a. Oh! Le facteur qui sonne et que personne n'entend !

b. Ah ! Mon livre que j'ai oublié ! Mes petites Heures en maroquin ! (Wehr, 1984 : 81, cité par Furukawa, $1996: 85)$

Il faut donc admettre que l'asymétrie sujet-objet n'est pas une contrainte syntaxique, comme le soutiennent Guasti (1992) et Cinque (1995), mais seulement une tendance qui dépend de facteurs pragmatiques. Furukawa (1996 : 6566) soutient en effet que l'objet peut être focalisé dans cette construction si son référent est plus saillant que le référent du sujet. En tout cas, l'existence de contre-exemples comme (Ia,b) n'invalide pas l'observation que l'asymétrie sujet-objet est par défaut manifestée dans la construction à focus phonologiquement emphatique de l'anglais, ainsi que dans les phrases nominales événementielles du français.

${ }^{23}$ Potts (2005: 7) soutient que la valeur affective est due à la sémantique lexicale. Il semble d'autre part difficile de spécifier l'item lexical associé à la valeur affective dans les phrases nominales événementielles. Il vaudrait la peine d'examiner si une construction syntaxique peut être le lieu d'une implicature conventionnelle, ce qui sera le sujet d'une étude ultérieure. 